\title{
An Observation Concerning Ritz-Galerkin Methods with Indefinite Bilinear Forms
}

\author{
By Alfred H. Schatz
}

\begin{abstract}
Existence, uniqueness and error estimates for Ritz-Galerkin methods are discussed in the case where the associated bilinear form satisfies a Garding type inequality, i.e., it is indefinite in a certain way. An application to the finite element method is given.
\end{abstract}

In this note, we would like to discuss existence, uniqueness and estimates over the whole domain for some Ritz-Galerkin methods where the bilinear form satisfies a Garding type inequality, i.e., it is indefinite in a special way. We shall first illustrate the problem by an example.

For simplicity, let $\Omega$ be a simply connected convex region in the plane with a polygonal boundary $\partial \Omega$ and consider the Dirichlet problem

(1) $L u=\sum_{i, j=1}^{2}-D_{i}\left(a_{i j}(x) D_{j} u\right)+\sum_{i=1}^{2} b_{i}(x) D_{i} u+c(x) u=f$ in $\Omega, \quad u=0$ on $\partial \Omega$,

where $L$ is uniformly elliptic in $\Omega$; for simplicity, we assume that the coefficients belong to $C^{1}(\bar{\Omega})$. Let us suppose that for each $f \in L_{2}(\Omega)$, the problem (1) has a unique solution $u$. It is then well known that $u \in \mathscr{W}_{2}^{1}(\Omega) \cap W_{2}^{2}(\Omega)$. Suppose that we wish to approximate $u$ using the finite element method. For this, we subdivide $\Omega$ into triangles with largest side $h$ and smallest angle $a \geqslant a_{0}>0$ and define a finite-dimensional subspace $S^{h} \subset \stackrel{\circ}{W}_{2}^{1}$ to be, for example, the set of piecewise linear functions on this triangulation which vanish on $\partial \Omega$. We then seek to determine an approximate solution $u^{h} \in S^{h}$ from the Ritz-Galerkin equations

$$
\begin{aligned}
B\left(u^{h}, \varphi\right) & =B(u, \varphi)=\iint_{\Omega} f \varphi d x, \quad \text { for all } \varphi \in S^{h}, \\
B(u, \varphi) & =\iint_{\Omega}\left(\sum_{i, j=1}^{2} a_{i j}\left(D_{i} u\right)\left(D_{j} \varphi\right)+\sum_{i=1}^{2} b_{i}\left(D_{i} u\right) \varphi+c u \varphi\right) d x .
\end{aligned}
$$

Let us note the inequality

$$
|B(u, \varphi)| \leqslant C\|u\|_{1}\|\varphi\|_{1}, \quad \text { for all } u, \varphi \in \stackrel{\circ}{W}_{2}^{1}(\Omega) .
$$

Received October 15, 1973.

AMS (MOS) subject classifications (1970). Primary 65N30, 65N 15.

Copyright $\odot$ 1974, American Mathematical Society 
In general, we have Gårding's inequality

$$
C_{1}\|v\|_{1}^{2}-C_{2}\|v\|_{0}^{2} \leqslant|B(v, v)| \quad \text { for all } v \in \stackrel{\circ}{W_{2}^{1}}(\Omega) .
$$

Here, $C_{1}$ and $C_{2}$ are constants with $C_{1}>0$ and $\|\cdot\|_{s}$ denotes the norm on the Sobolev space $W_{2}^{s}(\Omega)$ with $W_{2}^{0}(\Omega)=L_{2}(\Omega)$.

In the case that $C_{2} \leqslant 0$, and hence $B$ is positive definite, the existence and uniqueness of $u^{h}$ satisfying (2) follows from (3). This case has been extensively studied in the literature (e.g., [2], [5]). If however $C_{2}>0$, then $B$ may be indefinite and the existence and uniqueness of $u^{h}$ does not immediately follow. In fact, simple examples show that a solution of (2) need not exist, depending on the subspace $S^{h}$ used. It will turn out however that, if $h$ is sufficiently small, then existence and uniqueness of $u^{h}$ can be guaranteed and quasi-optimal error estimates hold.

Let us show how this may be done very simply. We shall first derive an a priori estimate. If $e=u-u^{h}$ satisfies (2), then using (3) and (4) we obtain

Dividing through by $\|e\|_{1}$, we have

$$
\begin{aligned}
C_{1}\|e\|_{1}^{2}-C_{2}\|e\|_{0}^{2} & \leqslant|B(e, e)|=|B(e, u)| \\
& \leqslant C\|e\|_{1}\|u\|_{1} .
\end{aligned}
$$

$$
\|e\|_{1}-\left(C_{2} / C_{1}\right)\|i e\|_{0} \leqslant C / C_{1}\|u\|_{1} \text {. }
$$

Now, if $e=u-u^{h}$ satisfies (2), a very useful argument of Nitsche [4] can be used to show the a posteriori estimate

$$
\|e\|_{0} \leqslant C_{4} h\|e\|_{1}, \quad C_{4} \text { independent of } h \text { and } e .
$$

This inequality is derived, for example in [2], where the above example was discussed in the case that $C_{2}=0$, i.e., $B$ is positive definite. Essential use is made of the assumption that (1) and the associated adjoint problem are uniquely solvable.

Collecting (5) and (6) and taking $h<\left(C_{1} / C_{2}\right) C_{4}$, when $C_{2}>0$, we obtain

$$
\left\|u-u^{h}\right\|_{1} \leqslant C_{5}\|u\|_{1}, \quad C_{5} \text { independent of } h, u \text { and } u^{h} \text {. }
$$

Our result now follows easily. For if $u^{h}$ is a solution of (2) corresponding to $u=0$, it follows from (7) that $u^{h}=0$. Hence, the homogeneous equation has a unique solution and, since $S^{h}$ is finite dimensional, this implies that $u^{h}$ exists and is unique for each $u \in \stackrel{\circ}{W}_{2}^{1}(\Omega)$. The optimal error estimates

$$
\|e\|_{j} \leqslant C_{6} h^{s-j}\|u\|_{s}, \quad(j=0,1) \text { and }(s=1,2)
$$

follow in the usual way from (6), (7) and the properties of $S^{h}$. Let us remark that if the polygonal domain $\Omega$ is not convex, i.e., it has an interior angle which is 
greater than $\pi$, then one can show that instead of $(6)$ one has $\|e\|_{0} \leqslant C_{4} h^{\gamma}\|e\|_{1}$. Here, $\gamma$ is positive and depends on the maximum interior angle and the nature of the coefficients of $L$ in a neighborhood of the vertex of the corresponding corner. In this case, the proof of existence of $u^{h}$ is essentially the same, but quasi-optimal estimates in $L_{2}(\Omega)$ are not obtained.

Let us now show that the method outlined above may be extended to more general situations. We shall consider our problem in a setting which is a slightly modified version of a general formulation of Ritz-Galerkin methods given by Babuška (cf. [1], [2]).

Let $H_{1} \subseteq H_{0}$ be real Hilbert spaces with a continuous embedding; i.e., $\|u\|_{H_{0}} \leqslant\|u\|_{H_{1}}$ for all $u \in H_{1}$. Let $B(u, v)$ be a bilinear form defined on $H_{1} \times H_{1}$ and, for $h \in(0,1]$, let $S^{h}$ denote a one-parameter family of finite-dimensional subspaces of $H_{1}$. For given $u \in H_{1}$, we seek a solution $u^{h} \in S^{h}$ satisfying

$$
B\left(u-u^{h}, \varphi\right)=0, \text { for all } \varphi \in S^{h}
$$

We shall make the following assumptions concerning $B$ and $S^{h}$ :

A1. For any $\eta \in S^{h}$, there exist constants $C_{1}>0$ and $C_{2}$, both independent of $\eta$ and $h$, such that

$$
C_{1}\|\eta\|_{H_{1}}-C_{2}\|\eta\|_{H_{0}} \leqslant \sup |B(\eta, \varphi)|, \quad \text { for all } \varphi \in S^{h} \text { with }\|\varphi\|_{H_{1}}=1 \text {. }
$$

A2. There exists a constant $C$ such that

$$
|B(u, v)| \leqslant C\|u\|_{H_{1}}\|v\|_{H_{1}}, \quad \text { for all } u, v \in H_{1} .
$$

A3. The following analogue of (6) holds: There exists a function $w(h)$ such that, if $e=u-u^{h}$ is a solution of (9), then

$$
\|e\|_{H_{0}} \leqslant w(h)\|e\|_{H_{1}},
$$

where $\lim w(h)=0$ as $h \longrightarrow 0$.

THEOREM. Suppose that the above conditions hold. Then there exists an $h_{0}>0$ such that, for all $h \in\left(0, h_{0}\right], E q$. (9) has a unique solution $u^{h} \in S^{h}$ for each $u \in H_{1}$. Furthermore,

$$
\|e\|_{H_{1}} \leqslant C\|u\|_{H_{1}} \quad \text { and } \quad\|e\|_{H_{0}} \leqslant C w(h)\|u\|_{H_{1}},
$$

where $C$ is independent of $h$ and $u$.

Proof. We first remark that, if $C_{2} \leqslant 0$, then the result easily follows. Suppose $e=u-u^{h}$ satisfies (9). Then, from (10), we have for $\varphi \in S^{h}$, \|\|$_{H_{1}}=1$, that 


$$
C_{1}\left\|u^{h}\right\|_{H_{1}}-C_{2}\left\|u^{h}\right\|_{H_{0}} \leqslant \sup \left|B\left(u^{h}, \varphi\right)\right| \leqslant \sup |B(u, \varphi)| \leqslant C\|u\|_{H_{1}}
$$

or

$$
\left\|u^{h}\right\|_{H_{1}}-\left(C_{2} / C_{1}\right)\left\|u^{h}\right\|_{H_{0}} \leqslant\left(C / C_{1}\right)\|u\|_{H_{1}}
$$

Using the triangle inequality, we obtain

$$
\|e\|_{H_{1}}-\left(C_{2} / C_{1}\right)\|e\|_{H_{0}} \leqslant C\|u\|_{H_{1}}
$$

where $C$ is a new constant independent of $u, e$ and $h$. The inequality (14) is analogous to (5) and the proof now proceeds in the same way as in the example given except that we use (12) instead of (6).

The essential point here is that an inequality of the type (12) may be effectively used to treat indefinite bilinear forms where the indefiniteness is caused by lower order terms. For many methods, this inequality can be established using the previously mentioned technique of Nitsche.

Department of Mathematics

Cornell University

Ithaca, New York 14850

1. I. BABUŠKA, "Error-bounds for finite element method," Numer. Math., v. 16, 1971, pp. 322-333. MR 44 \#6166.

2. I. BABUŠKA, The Mathematical Foundations of the Finite Element Method, With Applications to Partial Differential Equations (edited by A. D. Aziz), Academic Press, New York and London, 1972.

3. J. BRAMBLE \& M. ZLAMAL, "Triangular elements in the finite element method," Math. Comp., v. 24, 1970, pp. 809-820. MR 43 \#8250.

4. J. NITSCHE, "Lineare Spline-Funktionen und die Methode von Ritz für elliptische Randwertprobleme," Arch. Rational Mech. Anal., v. 36, 1970, pp. 348-355. MR 40 \#8250.

5. M. ZLÁMAL, "A finite element procedure of the second order of accuracy," Numer. Math., v. 14, 1969/70, pp. 394-402. MR 41 \#1233. 